\title{
Near-Earth Wave Propagation Characteristics of Electric Dipole in Presence of Vegetation or Snow Layer
}

\author{
DaHan Liao, Student Member, IEEE, and Kamal Sarabandi, Fellow, IEEE
}

\begin{abstract}
The problem of near-earth wave propagation in the presence of a dielectric layer such as a vegetation or snow covering is considered in this paper by modeling the propagation environment as a homogeneous two-layer medium (air/dielectric/ground). A number of studies have demonstrated the relevancy of the lateral wave for the case when both the transmitter and receiver are located within a simple half-space dielectric medium [1]-[6]. Unfortunately, for the generalized two-layer model, for configurations in which the transmitter or receiver (or both) is located above the dielectric layer, far-field analytical expressions that include all propagation features do not exist. In this paper, in order to arrive at a computational efficient solution for the two-layer model, a secondorder asymptotic evaluation for the electric fields of an arbitrarily oriented, infinitesimal electric dipole-for source and observation points located in the vicinity of the air/dielectric interface-is carried out through the method of steepest descents. The formulations are valid in the far field, with the limitation that the exponentially decaying pole and branch cut contributions have been ignored. It is observed that the Norton wave, though it is highly localized near the air/dielectric interface, is a significant contribution either when the dipole and observation points are both located above the dielectric layer or when one is above and the other is within the layer.
\end{abstract}

Index Terms-Asymptotic evaluation, near-earth wave propagation, two-layer half-space medium.

\section{INTRODUCTION}

$\mathbf{T}$ HE rise in the number of deployment of unattended ground sensor networks for environmental and military surveillance applications has prompted the need for a more complete understanding of near-earth wireless communication channels. An unattended ground sensor (UGS) is a low-power and often low-profile wireless transceiver that gathers a variety of data (acoustic, seismic, meteorological, etc.) from its surrounding and then transmits this information to another node within the network or to a central processing station. Since the heights of the transmitters and receivers in the network are low, the effects of the ground and of any dielectric covering (i.e., vegetation or snow) have to be included for an accurate understanding of the propagation characteristics necessary for predicting node connectivity.

The asymptotic solution to the problem of dipole radiation in the presence of a vegetated-terrain at VHF was studied first

Manuscript received October 12, 2004; revised April 13, 2005. This work was supported by the U.S. Army Research Laboratory (CTA-Communications and Networks) through Telcordia Technologies under Contract Sub-K\#10085872.

The authors are with the Radiation Laboratory, Department of Electrical Engineering and Computer Science, The University of Michigan, Ann Arbor, MI 48109 USA (e-mail: liaod@umich.edu).

Digital Object Identifier 10.1109/TAP.2005.856347 by Tamir [3] and subsequently by others including Li et al. [4], Cavalcante and Giarola [5], and Sarabandi and Koh [6]. However, in all these studies, both the source and observation points are assumed to be embedded in the vegetation; for such a configuration, the far-field radiation is dominated by the lateral wave and the Norton wave is of little importance; for the generalized configuration, in which the source and observation point locations can be arbitrary (with respect to the air/dielectric interface), a compact and comprehensive set of formulations for computing the total field cannot be found in existing literature. The more general problem of dipole radiation in the presence of a layered media has been thoroughly treated, but the associated equations are in integrals of the Sommerfeld form and are too complicated for computation when the source and observation points are far apart. The transformation of these integrals into asymptotic forms has been discussed, for instance, by Chew and Kong [7] - for a general two-layer medium but only for source and observation points directly on the air/dielectric interface, and by Marin and Pathak [8] — for applications pertaining to grounded, lossless double-layer printed circuitry. While it is possible that the results generated by Marin and Pathak can be extended and specialized to the class of near-earth propagation problems treated in this paper, no attempts have been made toward such an endeavor; in addition, although their closed-form expressions have been shown to be valid even in the near-field region of the source, the equations are rather involved and cannot be implemented easily as a consequence of the existence of poles and branch cuts as well as the proximity of these singularities to the saddle point. Our objective in this paper is to present field expressions assembled by retaining only a dominant asymptotic contribution; for application to propagation problems in the far field (or, as it is in our case, when the transmitter and receiver are separated by hundreds or thousands of meters or even more), this simplification is justified and an accurate approximation to the far field radiation characteristics can be readily obtained in an efficient manner.

The present paper is an extension of a previous study [6], which investigated the propagation characteristics for the case when both the source and observation points are embedded inside a vegetation layer. In this paper, we broaden our analysis to the general case in which the locations of the source and observation points can be at any location above ground. Specifically, the following three configurations are analyzed in order: 1) source and observation points in air; 2) source in dielectric layer, observation point in air; 3) source and observation points in dielectric layer. The fields of other configurations can 


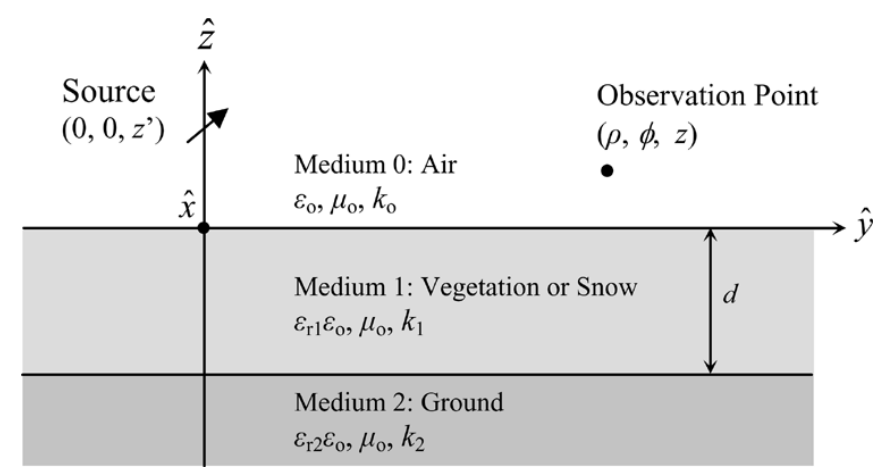

Fig. 1. Two-layer medium model for calculating the fields of an arbitrarily oriented electric dipole.

be easily deduced through the reciprocity principle. Since the third case has been thoroughly investigated by [6], only the results are presented here; for the complete derivation, the reader is referred to the original work.

The propagation of electromagnetic waves in the presence of vegetation or snow-covered terrain can be understood by considering vegetation or snow as a homogeneous layer with an effective dielectric constant and analyzing the fields in the resulting two-layer medium (Fig. 1). In order for this model to be accurate, especially in the case of a vegetation layer, the wavelength of propagation should be large compare to the dimensions of the features inside the layer (e.g., branches, leaves, spacing between individual plants). As suggested by [3] and [4], for a forest environment, the highest frequency at which the vegetation layer can be considered to be homogeneous is around $200 \mathrm{MHz}$; however, for short vegetation such as grass or crops, the effective medium theory is valid up to the frequency region of about 1 $\mathrm{GHz}$ before scattering from vegetation particles become significant. For a snow layer, the limit can be even extended to the millimeter-wave range due to low volume-scattering and the high degree of uniformity within the medium. The effective dielectric constants of the vegetation or snow layer and of the ground can be computed with the use of standard mixing models as described by [9] to [13].

From the spectral representation of the dyadic Green's function (as given by [14]), exact expressions for the fields of an infinitesimal electric dipole radiating in the presence of a twolayer medium can be derived. These expressions are listed in the Appendix. In Section II, asymptotic evaluations of these expressions in the most general from are carried out by applying the method of steepest descents. In Section III, simulation results are presented, and the variation of each component of the electric field is analyzed in terms of the effective permittivity, thickness of the vegetation or snow layer, locations of the dipole and observation points, and frequency of operation.

\section{AnAlytical Formulation}

In this section, analytical formulations for the calculation of the electric fields radiating from a short dipole of arbitrary orientation situated above a dielectric ground plane in the presence of a layer of vegetation or snow are derived. The geometry of the problem is illustrated in Fig. 1. As it has been noted, there are three primary configurations of interest, as a result of different combinations of the locations of the electric dipole and observation point. The dipole is assumed to be located on the $z$-axis with current moment vector $I_{o} \vec{l}=I_{o}\left(l_{x} \hat{x}+l_{y} \hat{y}+l_{z} \hat{z}\right)$, and the observation point-identified with the usual cylindrical coordinates-is at $(\rho, \phi, z)$. In the type of propagation problems considered here, for analysis restricted to the far field, it can be assumed that the magnitudes of $z^{\prime}, z$, and $d$ are much smaller than that of $\rho$; the radial distance between the dipole and the observation point. Also note that primed quantities are associated with the source, and the $\exp (i \omega t)$ convention is assumed and suppressed in all the formulations.

\section{A. Case $1\left(z^{\prime}>0\right.$ and $\left.z>0\right)$}

Consider the simple case when the source and observation points are both located in the upper layer (air) of a one-layer medium (Fig. 1, disregarding the last layer for the moment), the total electric field for a vertical dipole can be written as

$$
E_{x, y, z}^{\text {total }}=E_{x, y, z}^{\text {direct }}+E_{x, y, z}^{\text {scattered }} .
$$

Specifically, at grazing angle $\left(z^{\prime} \ll \rho\right.$ and $\left.z \ll \rho\right)$, each component of the field can be expanded as follows:

$$
\begin{aligned}
E_{z}^{\mathrm{total}}= & \frac{i \omega u_{o} I_{o} l_{z} e^{i k_{o} \rho}}{4 \pi} \\
& \left\{\frac{1}{\rho}+\frac{R_{T M}}{\rho}+\frac{C_{z, 1}}{\rho^{2}}+\frac{C_{z, 2}}{\rho^{3}} \cdots\right\} \\
E_{x}^{\mathrm{total}}= & \frac{i \omega u_{o} I_{o} l_{z} e^{i k_{o} \rho}}{4 \pi} \cos \phi \\
& \left\{\frac{\left(z^{\prime}-z\right)}{\rho^{2}}-\frac{\left(z^{\prime}+z\right) R_{T M}}{\rho^{2}}+\frac{C_{x, 1}}{\rho^{2}}+\frac{C_{x, 2}}{\rho^{3}} \cdots\right\} \\
E_{y}^{\mathrm{total}}= & \frac{i \omega u_{o} I_{o} l_{z} e^{i k_{o} \rho}}{4 \pi} \sin \phi \\
& \left\{\frac{\left(z^{\prime}-z\right)}{\rho^{2}}-\frac{\left(z^{\prime}+z\right) R_{T M}}{\rho^{2}}+\frac{C_{y, 1}}{\rho^{2}}+\frac{C_{y, 2}}{\rho^{3}} \cdots\right\} .
\end{aligned}
$$

The first term in the brackets is the direct field; the second term is the geometrical-optics reflection field; and the rest of the terms accounts for higher order scattered fields-where the $C s$ denote unknown factors. Since incidence is near grazing angle, it is easy to show that $R_{T M} \approx-1,\left(1-R_{T M}\right) \approx 2$, whereas $\left(1+R_{T M}\right)$ is a small quantity proportional to $1 / \rho$. For the $z$-component of the field, it can be seen that the cancellation of the direct and geometrical-optics reflection term generates a $1 / \rho^{2}$ resultantwhich is of the same order as the next term in the expansion. Likewise, for the $x$ and $y$ components, the sum of the direct and geometrical-optics reflection also produces a term that is of the same order as the next higher order term. Similar analysis can be applied to a horizontal dipole. Thus, when the source and observation points are close to the interface (incidence at grazing angle), it is apparent that an accurate description of the field components necessitates the derivation of the higher order term that comes after the geometrical-optics reflection term.

The complete evaluation of the field quantities begins with the two-fold integral form of the dyadic Green's function. A change of variable is applied to obtain the integration in terms of 
$k_{\rho}$ and Bessel functions. In integral form, the resulting expressions-for which exact closed-form solutions do not exist-of the $x, y$, and $z$ components of the scattered field are given in the Appendix. Since numerical computation of these expressions is slow and formidable due to the presence of singularities and the highly oscillatory behavior of the Bessel function at large distance $\rho$, approximate asymptotic solutions are sought. In a standard procedure, Hankel's functions of the first and second kind are used in extending the limits of integration to negative and positive infinity (in $k_{\rho}$-plane). By transforming the integration over to the complex $w$-plane by the change of variable $k_{\rho}=k_{o} \sin w$, and then substituting the asymptotic form of the Hankel's function for large arguments, the integrand can be written in the following form - to which the standard method of steepest descents can be applied

$$
\begin{aligned}
E_{i j}^{s}(\rho, \phi, z)=\frac{-k_{o}^{3} \eta_{o} I_{o} l_{i}}{16 \pi} & \sqrt{\frac{2}{i k_{o} \rho \pi}} \\
& \cdot \int_{-(\pi / 2)+i \infty}^{(\pi / 2)-i \infty} f_{i j}(w) e^{q(w)} d w
\end{aligned}
$$

where subscripts $i, j=x, y$, or $z$ and $q(w)=i k_{o} R \cos (w-$ $\left.w_{s}\right)$. The saddle point at $w=w_{s}$ is defined by $q^{\prime}\left(w_{s}\right)=0$, therefore

$$
\begin{aligned}
\sin w_{s} & =\frac{\rho}{R} \\
\cos w_{s} & =\frac{z+z^{\prime}}{R} \\
R^{2} & =\rho^{2}+\left(z+z^{\prime}\right)^{2} .
\end{aligned}
$$

After some manipulations and following the procedure of saddle point integration provided by [2], the $j$ component of the saddle point contribution up to second order for a dipole pointed in the $i$ direction can be written as

$$
\begin{aligned}
E_{i j}^{s p}=\frac{-k_{o}^{3} \eta_{o} I_{o} l_{i}}{8 \pi} e^{i k_{o} \rho} \cdot\left\{f_{i j}\left(w_{s}\right)\left(\frac{1}{i k_{o} \rho}\right)\right. \\
\left.+\frac{1}{2}\left[f_{i j}^{\prime \prime}\left(w_{s}\right)+\frac{f_{i j}\left(w_{s}\right)}{4}\right]\left(\frac{1}{i k_{o} \rho}\right)^{2}\right\}
\end{aligned}
$$

where the two terms represent the first and second saddle point expansion terms. The first saddle point expansion term is the geometrical-optics reflection term and the second expansion term can be thought as a correction term that becomes dominant when the source and observation points are close to the interface. This correction term is also commonly known as the Norton wave [15]. After some lengthy algebraic manipulations, expressions for $f_{i j}(w)$ of (9) can be shown to be the following:

$$
\begin{aligned}
f_{z x} & =-\frac{2}{k_{o}} \sin ^{3 / 2} w \cos w R_{T M}^{01}(w) \cos \phi \\
f_{z y} & =-\frac{2}{k_{o}} \sin ^{3 / 2} w \cos w R_{T M}^{01}(w) \sin \phi \\
f_{z z} & =\frac{2}{k_{o}} \sin ^{5 / 2} w R_{T M}^{01}(w) \\
f_{x x, y y} & =\sin ^{1 / 2} w \cos w\left[R_{-}(w) \mp R_{+}(w) \cos (2 \phi)\right] \\
f_{x y, y x} & =-\sin ^{1 / 2} w \cos w R_{+}(w) \sin (2 \phi)
\end{aligned}
$$

$$
\begin{aligned}
& f_{x z}=\frac{2}{k_{o}} \sin ^{3 / 2} w \cos w R_{T M}^{01}(w) \cos \phi \\
& f_{y z}=\frac{2}{k_{o}} \sin ^{3 / 2} w \cos w R_{T M}^{01}(w) \sin \phi .
\end{aligned}
$$

The mixed reflection coefficient, which is required for an $x$ or $y$-directed dipole, is defined as

$$
R_{ \pm}(w)=\frac{R_{T E}^{01}(w)}{k_{o} \cos w} \pm \frac{\cos w R_{T M}^{01}(w)}{k_{o}} .
$$

The TE and TM reflection coefficients are of the standard form

$$
\begin{aligned}
R_{T E}^{m n}(w) & =\frac{\sqrt{\varepsilon_{r m}-\sin ^{2} w}-\sqrt{\varepsilon_{r n}-\sin ^{2} w}}{\sqrt{\varepsilon_{r m}-\sin ^{2} w}+\sqrt{\varepsilon_{r n}-\sin ^{2} w}} \\
R_{T M}^{m n}(w) & =\frac{\varepsilon_{r n} \sqrt{\varepsilon_{r m}-\sin ^{2} w}-\varepsilon_{r m} \sqrt{\varepsilon_{r n}-\sin ^{2} w}}{\varepsilon_{r n} \sqrt{\varepsilon_{r m}-\sin ^{2} w}+\varepsilon_{r m} \sqrt{\varepsilon_{r n}-\sin ^{2} w}} .
\end{aligned}
$$

The appearance of branch points at $w_{b}= \pm \sin ^{-1} \sqrt{\varepsilon_{r 1}}$ entails the use of a two-sheeted Riemann surface in representing the $w$-plane; in order to satisfy the radiation condition, the path of integration of (5) is restricted to lie on the upper sheet $\left(\operatorname{Im} \sqrt{\varepsilon_{r 1}-\sin ^{2} w}>0\right)$. The branch cut defined by $w_{b}=\sin ^{-1} \sqrt{\varepsilon_{r 1}}$ may be crossed by the steepest descent path and its contribution to the integral can be included by adding the following to the saddle point contribution [2]:

$$
\begin{aligned}
E_{i j}^{b}= & \left(\frac{-k_{o} \eta_{o} I_{o} l_{i}}{8 \pi}\right)\left(\sqrt{-2 i} e^{-(3 / 2) i \arg \left(-i k_{o} \sqrt{1-\varepsilon_{r 1}}\right)}\right) \\
& \cdot\left(\frac{e^{i k_{1} \rho+i k_{o} \sqrt{1-\varepsilon_{r 1}}\left(z+z^{\prime}\right)}}{\sqrt{\rho}\left|\rho \sqrt{1-\varepsilon_{r 1}}-\left(z+z^{\prime}\right) \sqrt{\varepsilon_{r 1}}\right|^{3 / 2}}\right) \\
& \cdot \lim _{w \rightarrow w_{b}} \sqrt{w-w_{b}} f_{i j}^{\prime}(w) .
\end{aligned}
$$

It can be readily verified [1], [2] that the poles of the integrand in (5) are not intercepted as the original integration path deforms to the steepest descent path. Depending on the permittivity of the lower medium $\left(\varepsilon_{r 1}\right)$, these poles (the zeros of the denominator of the reflection coefficients in (18) and (19)) may move into the vicinity of the saddle point; thus, the poles may come into effect indirectly and must be taken into account by carrying out a modified saddle point integration method. Simulation results, however, show that the ordinary saddle point integration as used here is sufficient for predicting the field contributions in the far field. The total field is then the sum of the direct, saddle point, and branch cut contributions

$$
E_{i j}=E_{i j}^{d}+E_{i j}^{s p}+U\left(w_{s}-\theta_{b}\right) E_{i j}^{b}
$$

where $\theta_{b}=\operatorname{Re}\left(w_{b}\right)-\cos ^{-1} \operatorname{sech}\left[\operatorname{Im}\left(w_{b}\right)\right]$, and $U(\cdot)$ is the Heaviside step function. Calculation of (20) can be quite involved, but note that the branch cut contribution decays exponentially with $\rho$ since the wave number $k_{1}$ is complex; therefore, at large $\rho$, the branch cut contribution can be considered as negligible.

For a two-layer medium, to account for higher order reflections that are transmitted into and then emerging from the di- 
electric layer, the total reflection coefficient is modified as follows [14]:

$$
\begin{array}{r}
R_{P}(w)=R_{P}^{01}+\sum_{u=1}^{\infty} T_{P}^{01}\left(R_{P}^{12}\right)^{u}\left(R_{P}^{10}\right)^{u-1} \\
\cdot T_{P}^{10} e^{i 2 u d k_{\diamond} \sqrt{\varepsilon_{r 1}-\sin ^{2} w}} .
\end{array}
$$

Using the relations

$$
R_{P}^{m n}=-R_{P}^{n m} \text { and } T_{P}^{m n}=1+R_{P}^{m n}
$$

equation (22) can be rewritten as

$$
R_{P}(w)=\frac{R_{P}^{01}+R_{P}^{12} e^{i 2 d k_{o} \sqrt{\varepsilon_{r 1}-\sin ^{2} w}}}{1+R_{P}^{01} R_{P}^{12} e^{i 2 d k_{o} \sqrt{\varepsilon_{r 1}-\sin ^{2} w}}} .
$$

The subscript " $P$ " is a place holder for the polarization, either TE or TM. The saddle point contribution for each higher-order reflection $(u=1,2, \ldots \infty)$ in the summation series of (22) can be evaluated by using (9) after replacing the reflection coefficient term in $f_{i j}(w)$ with $T_{P}^{01}\left(R_{P}^{12}\right)^{u}\left(R_{P}^{10}\right)^{u-1} T_{P}^{10} e^{i 2 u d k_{\circ} \sqrt{\varepsilon_{r 1}-\sin ^{2} w}}$. Note that the higher-order reflections lead to higher order poles for the terms in the summation series of (22); although they are still located outside of the steepest descent path, these poles are close enough that - at higher orders - they demand a more refined saddle point integration method even when $\rho$ is relatively large. In order to circumvent this difficulty, calculations are made by following the normal mode approach in which the total aggregate reflection coefficient in (24) is inserted into (10)-(16) as a whole, rather than as individuals in a series. This approach leads to satisfactory results in the far field even if we are ignoring the pole contributions-which are now supplied by the zeros of the denominator in (24) and must be located through numerical methods before their contributions can be included using a standard technique [2]. These pole contributions, depending on their locations on the complex plane, represent either distinct surface-wave modes or leaky modes - both of which become less significant as the distance between source and observation points increases [2], [16]. Also note that in the two-layer case there are branch points only at $w_{b}= \pm \sin ^{-1} \sqrt{\varepsilon_{r 2}}$ since the expression in (24) can be shown to be an even function of $\sqrt{\varepsilon_{r 1}-\sin ^{2} w}$. The branch point that can be intercepted is at $w_{b}=\sin ^{-1} \sqrt{\varepsilon_{r 2}}$; but since medium 2 (the ground layer) is highly lossy, the branch cut contribution falls off asymptotically according to $\sim e^{i k_{2} \rho}$ and, hence, rapidly becomes much smaller than the algebraically decaying saddle point contribution.

\section{B. Case $2\left(-d<z^{\prime}<0\right.$ and $\left.z>0\right)$}

Exact formulations for this case have been derived and can be found in the Appendix. Proceeding through the same procedure as before, the method of steepest descents is employed in obtaining the integral after transforming the integration to the $w$-plane. Multiple reflections occurring within the dielectric layer can be accommodated by defining the total transmission coefficient as the following:

$$
\begin{aligned}
T_{P}^{\mathrm{up}}(w) & =T_{P}^{10} \sum_{u=0}^{\infty}\left(R_{P}^{10} R_{P}^{12}\right)^{u} e^{i 2 u d k_{o} \sqrt{\varepsilon_{r 1}-\sin ^{2} w}} \\
& =\frac{T_{P}^{10}}{1+R_{P}^{01} R_{P}^{12} e^{i 2 d k_{o} \sqrt{\varepsilon_{r 1}-\sin ^{2} w}}}
\end{aligned}
$$

and

$$
T_{P}^{\text {down }}(w)=T_{P}^{\mathrm{up}} R_{P}^{12} .
$$

The definition of (26) differs from that of (25) in that the former expression applies to waves containing an initial bounce off the dielectric layer/ground interface. It can be shown that the transmitted field $E_{i j}$ for each order $u$ can be written as

$$
\begin{aligned}
E_{i j, u}(\rho, \phi, z)=\frac{-k_{o}^{3} \eta_{o} I_{o} l_{i}}{16 \pi} & \sqrt{\frac{2}{i k_{o} \rho \pi}} \\
\cdot & \int_{-(\pi / 2)+i \infty}^{(\pi / 2)-i \infty} f_{i j, u}(w) e^{q(w)} d w
\end{aligned}
$$

where

$$
q(w)=i k_{o}(\rho \sin w+z \cos w) \approx i k_{o} \rho \cos \left(w-w_{s}\right)
$$

and the saddle point can be approximately defined by

$$
\cos w_{s} \approx \frac{z}{\rho} .
$$

The set of $f_{i j, u}(w)$ for an arbitrarily oriented dipole can be shown to take the following forms:

$$
\begin{aligned}
f_{z x, u}= & -\frac{2}{k_{o}} \frac{\sin ^{3 / 2} w \cos ^{2} w}{\sqrt{\varepsilon_{r 1}-\sin ^{2} w}} \\
& \cdot \cos \phi\left\{T_{T M, u}^{\text {up }} e^{c_{\text {up }}(w)}+T_{T M, u}^{\text {down }} e^{c_{\text {down }}(w)}\right\} \\
f_{z y, u}= & -\frac{2}{k_{o}} \frac{\sin ^{3 / 2} w \cos ^{2} w}{\sqrt{\varepsilon_{r 1}-\sin ^{2} w}} \\
& \cdot \sin \phi\left\{T_{T M, u}^{\text {up }} e^{c_{\text {up }}(w)}+T_{T M, u}^{\text {down }} e^{c_{\text {down }}(w)}\right\} \\
f_{z z, u}= & \frac{2}{k_{o}} \frac{\sin ^{5 / 2} w \cos w}{\sqrt{\varepsilon_{r 1}-\sin ^{2} w}} \\
& \cdot\left\{T_{T M, u}^{\text {up }} e^{c_{\text {up }}(w)}+T_{T M, u}^{\text {down }} e^{c_{\text {down }}(w)}\right\} \\
f_{x x, u}= & \sin ^{1 / 2} w \cos w\left\{\left[T_{+}^{\text {up }}-T_{-}^{\text {up }} \cos (2 \phi)\right] e^{c_{\text {up }}(w)}\right. \\
& \left.+\left[T_{-}^{\text {down }}-T_{+}^{\text {down }} \cos (2 \phi)\right] e^{c_{\text {down }}(w)}\right\} \\
f_{y y, u}= & \sin ^{1 / 2} w \cos w\left\{\left[T_{+}^{\text {up }}+T_{-}^{\text {up }} \cos (2 \phi)\right] e^{c_{\text {up }}(w)}\right. \\
& \left.+\left[T_{-}^{\text {down }}+T_{+}^{\text {down }} \cos (2 \phi)\right] e^{c_{\text {down }}(w)}\right\} \\
f_{x y, u}= & f_{y x, u}=-\sin ^{1 / 2} w \cos w \sin (2 \phi) \\
& \cdot\left\{T_{-}^{\text {up }} e^{c_{\text {up }}(w)}+T_{+}^{\text {down }} e^{c_{\text {down }}(w)}\right\}
\end{aligned}
$$




$$
\begin{aligned}
f_{x z, u}= & -\frac{2}{k_{o}} \sin ^{3 / 2} w \cos w \cos \phi \\
& \cdot\left\{T_{T M, u}^{\text {up }} e^{c_{\text {up }}(w)}-T_{T M, u}^{\text {down }} e^{c_{\text {down }}(w)}\right\} \\
f_{y z, u}= & -\frac{2}{k_{o}} \sin ^{3 / 2} w \cos w \sin \phi \\
& \cdot\left\{T_{T M, u}^{\text {up }} e^{c_{\text {up }}(w)}-T_{T M, u}^{\text {down }} e^{c_{\text {down }}(w)}\right\}
\end{aligned}
$$

in which

$$
\begin{aligned}
c_{\text {up }}(w) & =i k_{o} \sqrt{\varepsilon_{r 1}-\sin ^{2} w}\left|z^{\prime}\right| \\
c_{\text {down }}(w) & =i k_{o} \sqrt{\varepsilon_{r 1}-\sin ^{2} w}\left(z^{\prime}+2 d\right) .
\end{aligned}
$$

The designations "up" and "down" differentiate waves that are initially propagating upward and downward from the source. The mixed transmission coefficients have been written in the following form:

$$
\begin{aligned}
T_{ \pm}^{\mathrm{up}}(w) & =\frac{T_{T E, u}^{\mathrm{up}}}{k_{o} \sqrt{\varepsilon_{r 1}-\sin ^{2} w}} \pm \frac{T_{T M, u}^{\mathrm{up}} \cos w}{k_{o}} \\
T_{ \pm}^{\text {down }}(w) & =\frac{T_{T E, u}^{\text {down }}}{k_{o} \sqrt{\varepsilon_{r 1}-\sin ^{2} w}} \pm \frac{T_{T M, u}^{\text {down }} \cos w}{k_{o}} .
\end{aligned}
$$

In the formulations above, $T_{P, u}^{\mathrm{up}}$ and $T_{P, u}^{\mathrm{down}}$ are the individual terms in the infinite series of (25) and (26), respectively. Instead of computing each order of transmission separately, the saddle point evaluation of (27) can be carried out using (9), as before, by means of the normal mode approach-in which now the aggregate transmission coefficients in (25) and (26) are substituted into $f_{i j}(w)$ as one term. The difficulty mainly lies in taking the double derivative of $f_{i j}(w)$, but this can be overcome with the help of a symbolic math software. When the source and observation points are located in the vicinity of the interface, it is seen that both the first and second term in the saddle point expansion fall off as $1 / \rho^{2}$; thus, as in Case 1 , both expansion terms are necessary for accurate representation of the total field.

It is seen that the sign of the term $\sqrt{\varepsilon_{r 1}-\sin ^{2} w}$ has no effect on the final result in computing the integral in (27); therefore, once again, the function $f_{i j}(w)$ is an even function of $\sqrt{\varepsilon_{r 1}-\sin ^{2} w}$ and the only branch points on the complex $w$-plane are attributed to the term $\sqrt{\varepsilon_{r 2}-\sin ^{2} w}$. (As a matter of fact, for general stratified media problems, in the normal mode approach, the branch points on the $k_{\rho}$ plane are supplied only by the first and last layer [17]; the branch points of the first layer can be eliminated - as it has been done here-by translating the calculation onto the $w$-plane after the change of variable $k_{\rho}=k_{o} \sin w$. Thus, on the $w$-plane, the only branch points remaining are due to the last layer in the stratification.) Similar to Case 1, calculations are much simplified by ignoring contributions from the branch cut and the poles [which are provided by the zeros of the denominator of (25) and (26)]; in the far field, this claim is justified since the saddle point contribution becomes the only dominant field component.

\section{Case $3\left(-d<z^{\prime}<0\right.$ and $\left.-d<z<0\right)$}

As it has been shown in [6], when both the source and observation points are located inside medium 2 , the direct and saddle point contributions decay exponentially as a function of the ra- dial distance $\rho$ since their propagation takes place in a lossy medium; the dominant contribution to the total field, as it turns out, comes from a branch cut contribution. Although a modal analysis can be used, a ray tracing approach provides a more insightful interpretation for this case. In the ray tracing approach, the effective reflection coefficient is expanded as a series before asymptotic evaluation is carried out separately for each term. In such an approach, both medium 1 and medium 2 would furnish branch cut contributions on the $w$-plane since now-for each individual order of reflection-the integrand in the field integral is no longer an even function of $\sqrt{\varepsilon_{r 1}-\sin ^{2} w}$. Discarding the branch cut contribution arising from the branch point at $w_{b}=\sin ^{-1} \sqrt{\varepsilon_{r 2}}$ for the reason aforementioned, the only relevant and significant contribution, in the far field, comes from the branch cut contribution due to the branch point at $w_{b}=$ $\sin ^{-1} \sqrt{\varepsilon_{r 1}}$. This branch cut contribution, which now no longer undergoes exponential decay, can be interpreted as a wave from the source that radiates upward to the dielectric/air interface at critical angle and then propagates along the interface (in air) before reaching the observation point at critical angle again. Since the majority of the propagation takes place in air, this field component-which has been labeled as the "lateral" wave-does not suffer the large path loss experience by the direct and saddle point contributions. Through standard branch cut integration techniques, the three lowest orders of lateral waves have been derived in [6] and are expressed in matrix form as the following $\left(z^{\prime}>z\right)$ :

$$
\begin{aligned}
\vec{E}^{b}= & \frac{-i \eta_{1}}{2 \pi\left(1-\frac{1}{\varepsilon_{r 1}}\right)^{1 / 4}} \frac{e^{i k_{o} \rho}}{\sqrt{\rho}} \\
& \cdot\left\{\frac{e^{i k_{1} \sqrt{1-1 / \varepsilon_{r 1}}}\left(\left|z^{\prime}\right|+|z|\right)}{\left[\sqrt{\frac{1}{\varepsilon_{r 1}}}\left(\left|z^{\prime}\right|+|z|\right)-\rho \sqrt{1-\frac{1}{\varepsilon_{r 1}}}\right]^{3 / 2}} \overline{\bar{A}}\right. \\
& +\frac{e^{i k_{1}} \sqrt{1-1 / \varepsilon_{r 1}}\left(2 d-\left|z^{\prime}\right|+|z|\right)}{\left[\sqrt{\frac{1}{\varepsilon_{r 1}}}\left(2 d-\left|z^{\prime}\right|+|z|\right)-\rho \sqrt{1-\frac{1}{\varepsilon_{r 1}}}\right]^{3 / 2}} \overline{\bar{A}} \cdot \overline{\bar{R}} \\
& \left.+\frac{e^{i k_{1} \sqrt{1-1 / \varepsilon_{r 1}}\left(2 d+\left|z^{\prime}\right|-|z|\right)}}{\left[\sqrt{\frac{1}{\varepsilon_{r 1}}}\left(2 d+\left|z^{\prime}\right|-|z|\right)-\rho \sqrt{1-\frac{1}{\varepsilon_{r 1}}}\right]^{3 / 2}} \overline{\bar{A}} \cdot \overline{\bar{R}}\right\} \\
& \cdot I_{o} \vec{l}
\end{aligned}
$$

where $\overline{\bar{A}}$ and $\overline{\bar{R}}$ are symmetric dyads defined in [6]. The first term in (42) represents the direct lateral wave contribution; the second term is the contribution of the lateral wave generated from the image of the source in the ground plane; and the third term is the contribution of the direct lateral wave that has been reflected from the ground plane before reaching the observation point. It is seen that the lateral waves, and hence the total field, decreases as $1 / \rho^{2}$-which is the same asymptotic behavior observed for the first two cases. For further details on the derivation and verification of (42), the reader is referred to [6].

\section{Simulation RESULTS}

In this section, the field components for a vertical dipole and a horizontal dipole are calculated using the formulations derived 

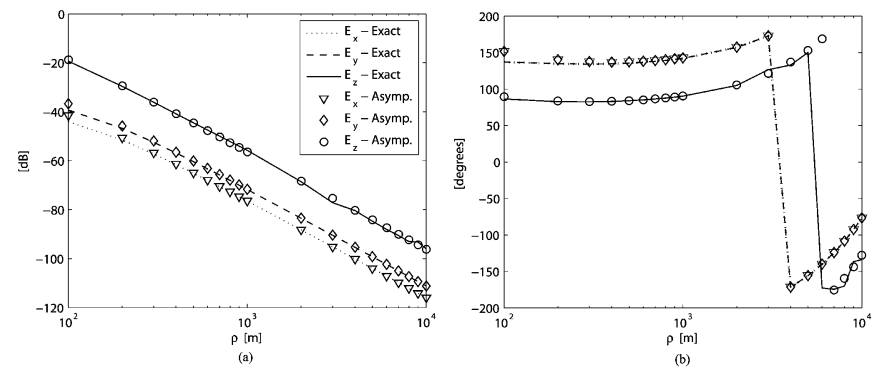

Fig. 2. Magnitude (a) and phase (b) of the electric fields of a vertical dipole as a function of distance in the presence of a vegetation layer with $d=0.5 \lambda$. The dipole is located on $z$-axis at $z^{\prime}=0.4 \lambda$ and the observation point is at $(\rho, \pi / 3,0.3 \lambda) ; f=30 \mathrm{MHz}, \varepsilon_{r 1}=1.01+0.01 i$, and $\varepsilon_{r 2}=8+6 i$.
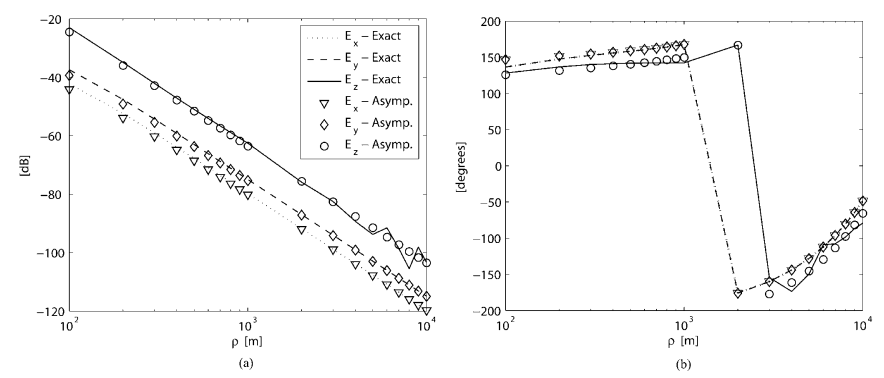

Fig. 3. Magnitude (a) and phase (b) of the electric fields of a vertical dipole as a function of distance in the presence of a dry snow layer with $d=0.5 \lambda$. The dipole is located on $z$-axis at $z^{\prime}=0.4 \lambda$ and the observation point is at $(\rho, \pi / 3,0.3 \lambda) ; f=30 \mathrm{MHz}, \varepsilon_{r 1}=2.01+0.01 i$, and $\varepsilon_{r 2}=8+6 i$. Note the poor quality of the numerical calculation for $E_{z}$ at large distances, but the asymptotic solution is well-behaved.

in Section II. The variation in radiation characteristics is also demonstrated as a function of the effective permittivities, thickness of the vegetation or snow layer, locations of the source and observation points, and frequency of operation. Before the analysis, it is necessary to verify the validity of the asymptotic expressions presented. Figs. 2 and 3 show the total fields (scattered plus direct) of a vertical dipole of unity current moment operating at $30 \mathrm{MHz}$ located on the $z$-axis at $z^{\prime}=0.4 \lambda$ with the observation point at $(\rho, \pi / 3,0.3 \lambda)$. The middle dielectric layer has been chosen to have a thickness of $0.5 \lambda$; the effective permittivity of the ground, $\varepsilon_{r 2}$, is set to be $8+6 i$, which approximately corresponds to a soil composed of gray San Antonio clay loam with a density of $1.4 \mathrm{~g} / \mathrm{cm}^{3}$ and $5 \%$ moisture content [12]. The dielectric layer is assumed to be sparse vegetation $\left(\varepsilon_{r 1}=1.01+0.01 i\right)$ in Fig. 2 and dry snow with density of 0.5 $\mathrm{g} / \mathrm{cm}^{3}\left(\varepsilon_{r 1}=2.01+0.01 i\right)$ in Fig. 3. Note the $1 / \rho^{2}$ dependence of all the field components in the far field. The exact solutions are obtained through numerical integration of the expressions tabulated in the Appendix. Figs. 4 and 5 show the fields for a horizontal dipole calculated using the same set of parameters, except now the dipole itself is located inside the dielectric layer at $z^{\prime}=-0.4 \lambda$. It can be seen that there is very good agreement (both in magnitude and phase) between the exact and asymptotically evaluated values when $\rho$ is large in all the cases. As it has been noted in Section II, the validity of the formulations presented here depends on the assumption that exponentially decaying components of the field can be discarded when $\rho$ is large. For example, in Fig. 5, the validity holds for $\rho \geq 30 \lambda$ below
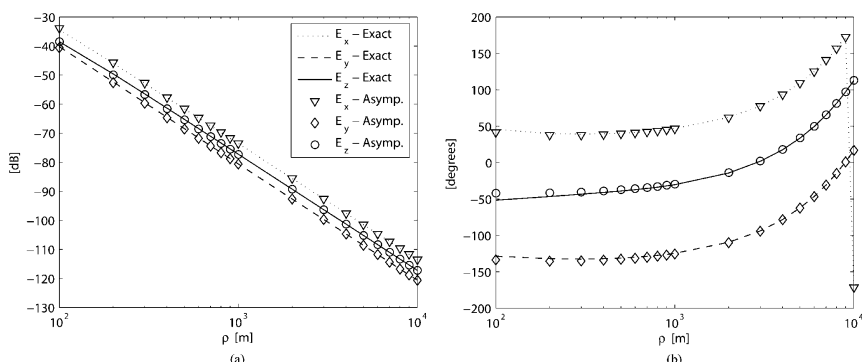

Fig. 4. Magnitude (a) and phase (b) of the electric fields of a horizontal dipole as a function of distance in the presence of a vegetation layer with $d=0.5 \lambda$. The dipole is located on $z$-axis at $z^{\prime}=-0.4 \lambda$ and the observation point is at $(\rho, \pi / 3,0.3 \lambda) ; f=30 \mathrm{MHz}, \varepsilon_{r 1}=1.01+0.01 i$, and $\varepsilon_{r 2}=8+6 i$.
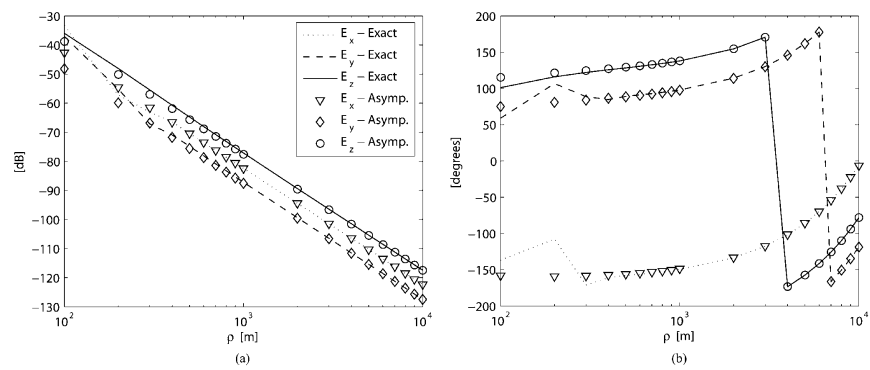

Fig. 5. Magnitude (a) and phase (b) of the electric fields of a horizontal dipole as a function of distance in the presence of a dry snow layer with $d=0.5 \lambda$. The dipole is located on $z$-axis at $z^{\prime}=-0.4 \lambda$ and the observation point is at $(\rho, \pi / 3,0.3 \lambda) ; f=30 \mathrm{MHz}, \varepsilon_{r 1}=2.01+0.01 i, \varepsilon_{r 2}=8+6 i$.
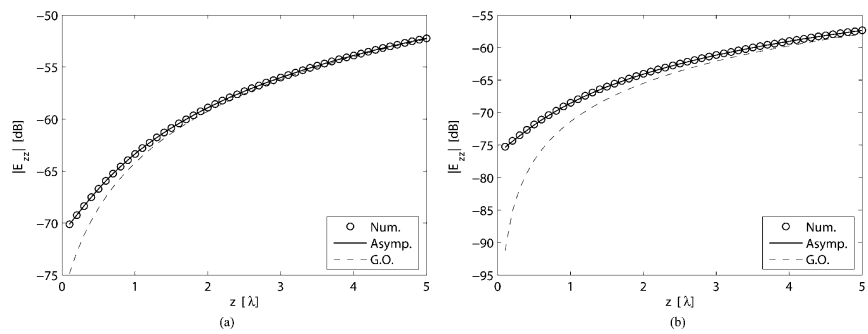

Fig. 6. Comparison of asymptotic and geometrical-optics approximations with exact values obtained from numerical integration for a vertical dipole located at $z^{\prime}=0.4 \lambda$ (a) and $z^{\prime}=-0.4 \lambda$ (b). Observation point is at $(200 \lambda, \pi / 3, z)$; $f=30 \mathrm{MHz}, \varepsilon_{r 1}=1.01+0.01 i, \varepsilon_{r 2}=8+6 i$, and $d=0.5 \lambda$.

which the branch cut and pole contributions must be included to accurately account for the total field, as well as the interference patterns.

Having validated the accuracy of the asymptotic expressions, it is beneficial to investigate the region in which the Norton wave [or the second term in (9)] is dominant. It is expected that when the source or observation point (or both) is far from the dielectric/air interface, the geometrical-optics term would become the principal field contribution. Depending on the configuration and physical parameters of the problem, simulations confirm that geometrical-optics provides an accurate approximation as long as the source or observation point (or both) is greater than $2 \lambda-3 \lambda$ above the dielectric layer. A comparison of the asymptotic and geometrical-optics approximations with the exact solutions is shown in Fig. 6(a) and (b) for a vertical dipole as a function of the observation point's distance above the dielectric. The dipole's location is in air for the first figure but is in the dielectric for the second. Note that the geometric-optics term converges to 


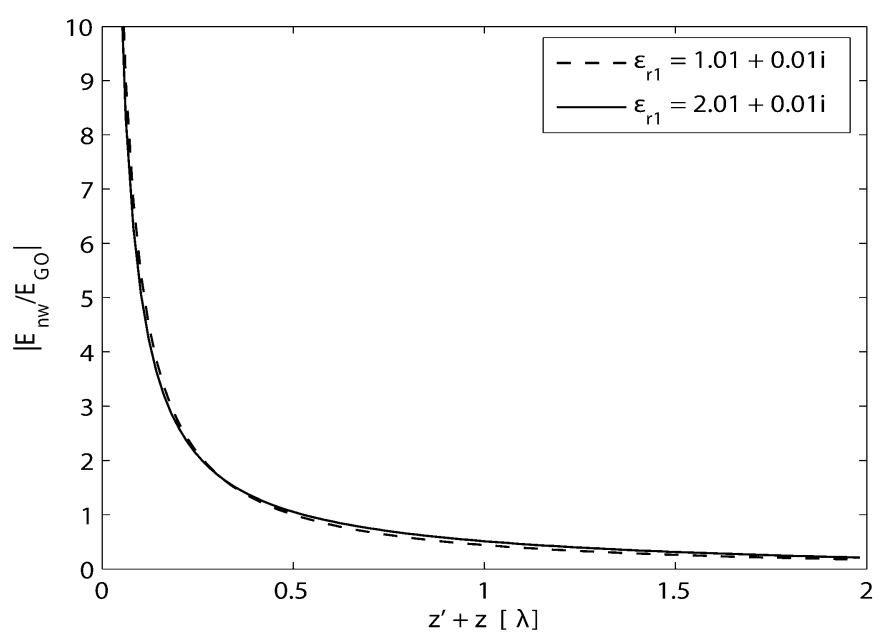

(a)

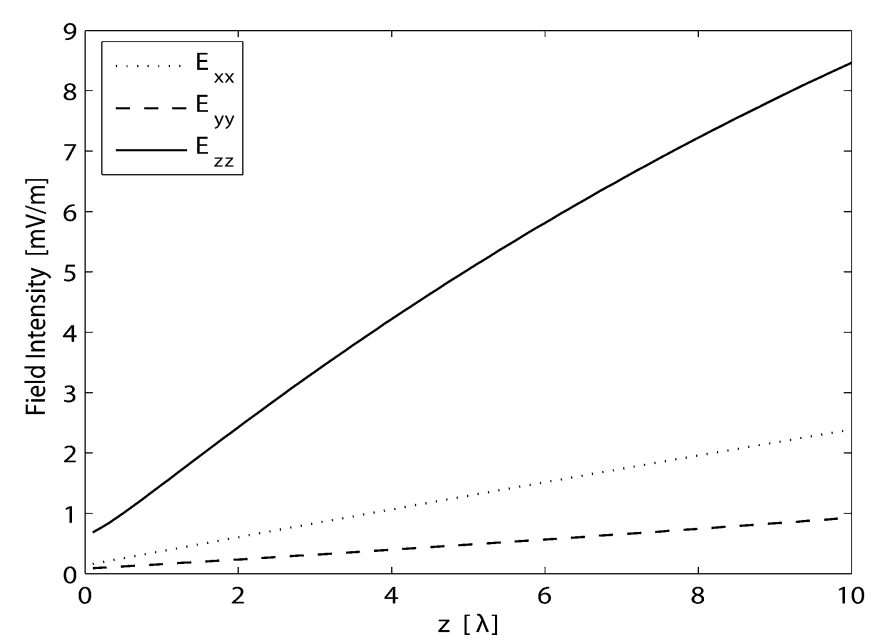

(b)

Fig. 7. (a) Ratio of Norton wave to G.O. contribution for a vertical dipole. Source and observation points located above dielectric layer; $f=30 \mathrm{MHz}, \rho=200 \lambda$, $\varepsilon_{r 2}=8+6 i$, and $d=0.5 \lambda$. (b) Field intensity as a function of receiver height above dielectric/air interface for a dipole located inside the dielectric at $z^{\prime}=-0.4 \lambda$. Observation point is at $(100 \lambda, \pi / 3, z) ; f=30 \mathrm{MHz}, \varepsilon_{r 1}=1.01+0.01 i, \varepsilon_{r 2}=8+6 i$, and $d=0.5 \lambda$.

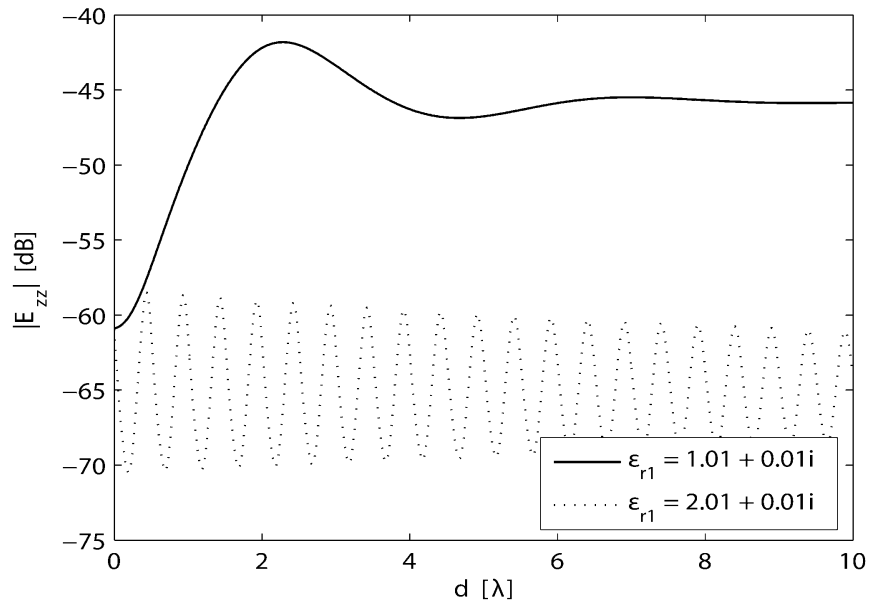

(a)

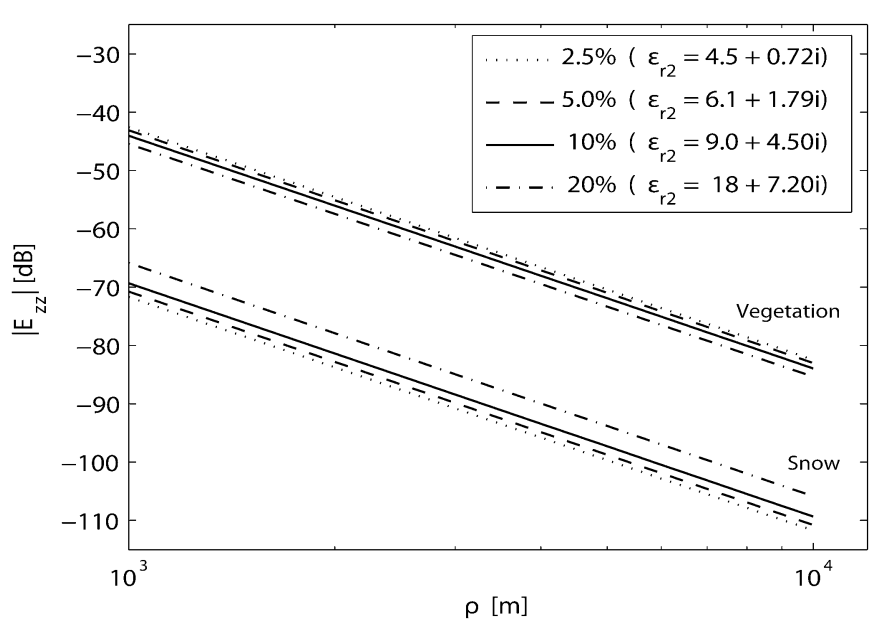

(b)

Fig.8. (a) Magnitude of $E_{z}$ for a vertical dipole located above a dielectric layer with $\varepsilon_{r 1}=1.01+0.01 i$ and $2.01+0.01 i$. Observation point is at $(100 \lambda, \pi / 3,0.3 \lambda) ; f=30 \mathrm{MHz}$ and $\varepsilon_{r 2}=8+6 i$. (b) Magnitude of $E_{z}$ for a vertical dipole in the presence of a ground with various percentages of soil moisture content. The dipole is located inside the dielectric layer at $z^{\prime}=-0.5 \lambda$ and observation point is at $(\rho, \pi / 3,0.5 \lambda) ; f=200 \mathrm{MHz}, \varepsilon_{r 1}=1.01+0.01 i$ for vegetation layer, $\varepsilon_{r 1}=2.01+0.01 i$ for snow layer, and $d=2.0 \lambda$.

the exact solution for large $z$ but becomes a rather poor approximation as the observation point approaches the interface. An example of the relative magnitude of the Norton wave as compared to the geometrical-optics approximation is shown in Fig. 7(a); the Norton wave is seen to be highly localized to the air/dielectric interface, hence, it is obvious why this wave contribution has been designated as a type of "surface" wave. From Fig. 6, one can also see that the field intensity at the receiver can be increased by elevating the receiver higher above ground, this is often called the height-gain effect. In fact, for small elevations, the field intensity increases linearly with elevation, as shown in Fig. 7(b), for a dipole embedded in the dielectric layer-a similar plot can be constructed for a dipole located in air.

It is also instructive to analyze how the field changes as a function of the thickness of the dielectric layer. As the thickness changes, interference patterns caused by the superposition of multiple reflections are observed; this is shown in Fig. 8(a) for a vertical dipole located above a dielectric layer with $\varepsilon_{r 1}=$ $1.01+0.01 i$ and $2.01+0.01 i$. The observation point is fixed in the far field at $(100 \lambda, \pi / 3,0.3 \lambda)$ in both cases. It can be deduced that the magnitude of the field oscillates about the limit value for which $d \rightarrow \infty$ (one-layer medium) and the rate of oscillation is dependent upon the magnitude of the permittivity whereas the rate of the approach toward the limit value is determined by the loss tangent.

To fully consider the effects of the dielectric layer and the ground, the total field's dependence on the effective permittivities of the two media must also be examined. For a vegetation layer, the effective dielectric constant is slightly greater than that of free space; on the other hand, for a snow layer, while the imaginary part of the permittivity is still small, the real part can be as large as 2.0-3.0, depending on the snow density and the moisture content. The field variations as a function of $\varepsilon_{r 1}$ for fixed dipole and observation point locations are shown in 

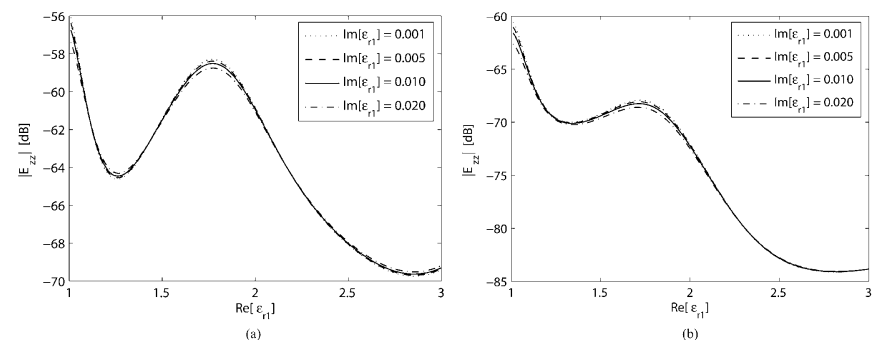

Fig. 9. Electric field as a function of the effective permittivity of the dielectric layer for a vertical dipole. Dipole is located above the dielectric layer for (a) and within the dielectric layer for (b). Observation point is at $(100 \lambda, \pi / 3,0.3 \lambda)$, $f=30 \mathrm{MHz}, \varepsilon_{r 2}=8+6 i$, and $d=0.5 \lambda$.

Fig. 9. Note that the field strength can change considerably as a function of the real part of the permittivity; and, for the set of parameters used in this example, there can be a 15 to $20 \mathrm{~dB}$ difference between the field value of a vegetation layer and that of a snow layer. The complex semi-oscillatory behavior evident in Fig. 9 can also be anticipated if the permittivity of the ground, $\varepsilon_{r 2}$, is varied while holding that of the dielectric layer constant. A primary determinant of the permittivity of soil is the moisture content. In Fig. 8(b), the effect of the moisture content on the total field is illustrated for a ground composed of gray San Antonio clay loam with a dry density of $1.4 \mathrm{~g} / \mathrm{cm}^{3}$; the value of the corresponding dielectric constant for each moisture level is obtained from the measurements done by Hipp [12]. It is obvious that the field strength can change quite noticeably as a function of the permittivity of the ground; in addition, it is interesting to note that the field strength, as the soil moisture content is elevated, decreases for a vegetation layer but increases for a snow layer.

To illustrate the frequency response of the present problem, a frequency dependent model must be used for the permittivities of the dielectric layer and ground. For the simulations that follow, the soil of the ground is again selected to be gray San Antonio clay loam with a density of $1.4 \mathrm{~g} / \mathrm{cm}^{3}$ (5\% moisture content). The dielectric constant as a function of frequency of such a composition can be found in figures given by [12]. For a vegetation layer, the effective dielectric constant is calculated by using the Polder-van Santen mixing formula, in which the host material is air and the inclusion being the vegetation material. The dielectric properties of the vegetation material are dependent upon the gravimetric moisture content, frequency, and temperature; an empirical formula in terms of these parameters has been derived in [10]. For a snow layer, if the snow is dry, then the real part of the effective dielectric constant can be considered to be independent of frequency and to be a function of the snow density only; the imaginary part is relatively small and on the order of $10^{-2}$ to $10^{-3}$.

The results of the simulations are shown in Fig. 10(a) and (b), the former is for a vegetation layer and the latter is for a dry snow layer; a ground with the soil parameters given above is used in both cases. The source is a vertical dipole located in air-two observation points are used: in air and in the vegetation or snow, hence, there are two graphs in each figure. In the frequency range of $30 \mathrm{MHz}$ to $200 \mathrm{MHz}$, the relative dielectric constant of soil changes from $8.10+6.00 i$ to $6.10+1.79 i$,
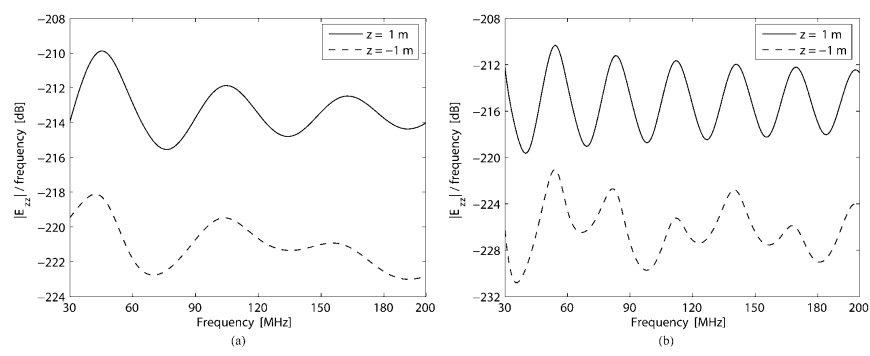

Fig. 10. Frequency response for a vertical dipole in a vegetation layer (a) and a snow layer (b). Dipole is located above the dielectric layer at $z^{\prime}=1 \mathrm{~m}$, observation point is at $(1000 \mathrm{~m}, \pi / 3, \pm 1 \mathrm{~m})$, and $d=2 \mathrm{~m}$.

and that of the vegetation layer changes from $1.09+0.001 i$ to $1.10+0.009 i$ for $5 \%$ inclusion by volume and for a vegetation material at $27^{\circ} \mathrm{C}$ with $50 \%$ gravimetric moisture content. For the dry snow, with a density of $0.5 \mathrm{~g} / \mathrm{cm}^{3}$, the relative dielectric constant is $\sim 2.01+0.01 i$ and is almost independent of frequency. Note that only the $z$-component of the electrical field is shown in the graphs, and the quantity has been normalized to frequency in order to set the power output of the transmitter to constant. As it is evident in these figures, the amplitudes of variation can be quite large; thus, in the design of low-power communication channels, the selected frequency of operation is a critical factor in determining the efficiency of the system.

\section{CONCLUSION}

In this study, the concept and relevance of Norton waves and lateral waves have been analyzed for the canonical problem of propagation in the presence of a two-layer medium representing a vegetation or snow-covered terrain. Through the method of steepest descents, asymptotic formulations for the total field of an arbitrarily oriented electric dipole have been derived from exact expressions for dipole and observation point situated near the air/dielectric interface. By discarding the pole and branch cut contributions for configurations in which the dipole and observation point both are not embedded within the dielectric layer, the formulations are much simplified and reduce to an ordinary saddle point contribution. It should be emphasized that such a simplification can only be made when there is loss in the media of propagation. In the absence of loss, in order to be considered as an accurate and practical solution, the saddle point contribution must be supplemented with the proper branch cut contributions-regardless of the magnitude of the radial distance between the transmitter and receiver. The pole contributions, however, would always decay exponentially as a function of radial distance even in the lossless case. When the aforementioned simplification is exploited, it has been shown that the saddle point contribution becomes the dominant field component and it is composed of a geometrical-optics term and a Norton wave correction term that is highly localized to the air/dielectric interface. In addition, simulation results indicated that the field intensity and frequency response at the receiver have a strong dependence on the permittivities of the vegetation or snow layer and the ground. Although the transmitter has been restricted to an electric dipole throughout this paper, extension to an arbitrary radiating source can be made by noting that the asymptotic form of the Green's function for each of the three cases discussed is 
related to the derived expressions for the field components by a simple constant:

$$
\overline{\bar{G}}\left(\vec{r}^{\prime}, \vec{r}\right)=\frac{1}{i \omega \mu_{o}}\left[\begin{array}{lll}
E_{x x} & E_{y x} & E_{z x} \\
E_{x y} & E_{y y} & E_{z y} \\
E_{x z} & E_{y z} & E_{z z}
\end{array}\right] .
$$

Therefore, once the current distribution of an arbitrary source is known, an approximation to the far field pattern can be easily computed. Also, upon application of the reciprocity principle, it is straightforward to verify that

$$
\overline{\bar{G}}\left(\vec{r}, \vec{r}^{\prime}\right)=\overline{\bar{G}}\left(\vec{r}^{\prime}, \vec{r}\right)^{T}
$$

where the superscript " $T$ " indicates the transpose operation. Simply by using the relation stated in (44), we can extend our formulations to other standard configurations not explicitly treated in Section II. For example, the dipole is located in air while the observation point is located inside the dielectric layer.

\section{APPENDIX I}

INTEGRAL SOLUTIONS FOR CASE $1\left(z^{\prime}>0\right.$ AND $\left.z>0\right)$

Exact expressions for the electric field of a dipole radiating in the presence of a two-layer medium are derived from the dyadic Green's function. If the arbitrarily oriented dipole is located on the $z$-axis at $\left(0,0, z^{\prime}\right)$ with the current moment vector $I_{o} \vec{l}=I_{o}\left(l_{x} \hat{x}+l_{y} \hat{y}+l_{z} \hat{z}\right)$, it can be shown that, for Case 1 , the general equation for the scattered electric field at observation point $(\rho, \phi, z)$ with $z^{\prime}>z$ can be written as

$$
E_{i j}^{s}=-\frac{\omega \mu_{o} I_{o} l_{i}}{8 \pi} \int_{0}^{\infty} f_{i j}\left(k_{\rho}\right) e^{i k_{o z}\left(z+z^{\prime}\right)} d k_{\rho}
$$

where the function in the integrand is dependent upon the dipole orientation and the field component of interest $(x, y$, or $z)$

$$
\begin{aligned}
& f_{z x}\left(k_{\rho}\right)=\frac{-2 i k_{\rho}^{2}}{k_{o}^{2}} R_{T M}\left(k_{\rho}\right) J_{1}\left(k_{\rho} \rho\right) \cos \phi \\
& f_{z y}\left(k_{\rho}\right)=\frac{-2 i k_{\rho}^{2}}{k_{o}^{2}} R_{T M}\left(k_{\rho}\right) J_{1}\left(k_{\rho} \rho\right) \sin \phi \\
& f_{z z}\left(k_{\rho}\right)=\frac{2 k_{\rho}^{3}}{k_{o z} k_{o}^{2}} R_{T M}\left(k_{\rho}\right) J_{0}\left(k_{\rho} \rho\right) \\
& f_{x x, y y}\left(k_{\rho}\right)=k_{\rho}\left[R_{-}\left(k_{\rho}\right) J_{0}\left(k_{\rho} \rho\right) \pm R_{+}\left(k_{\rho}\right) J_{2}\left(k_{\rho} \rho\right) \cos (2 \phi)\right] \\
& f_{x y, y x}\left(k_{\rho}\right)=k_{\rho} R_{+}\left(k_{\rho}\right) J_{2}\left(k_{\rho} \rho\right) \sin (2 \phi) \\
& f_{x z}\left(k_{\rho}\right)=\frac{2 i k_{\rho}^{2}}{k_{o}^{2}} R_{T M}\left(k_{\rho}\right) J_{1}\left(k_{\rho} \rho\right) \cos \phi \\
& f_{y z}\left(k_{\rho}\right)=\frac{2 i k_{\rho}^{2}}{k_{o}^{2}} R_{T M}\left(k_{\rho}\right) J_{1}\left(k_{\rho} \rho\right) \sin \phi \\
& \text { The total reflection } \operatorname{coefficient} \text { is given by } \\
& R_{P}=R_{P}^{01}+T_{P}^{01} T_{P}^{10} \sum_{u=1}^{\infty}\left(R_{P}^{10}\right)^{u-1}\left(R_{P}^{12} e^{i 2 k_{1 z} d}\right)^{u} \\
&=R_{P}^{01}+\frac{\left[1-\left(R_{P}^{01}\right)^{2}\right] R_{P}^{12} e^{i 2 k_{1 z} d}}{1+R_{P}^{01} R_{P}^{12} e^{i 2 k_{1 z} d}} \\
&=\frac{R_{P}^{01}+R_{P}^{12} e^{i 2 k_{1 z} d}}{1+R_{P}^{01} R_{P}^{12} e^{i 2 k_{1 z} d}}
\end{aligned}
$$

and the mixed reflection coefficient is

$$
R_{ \pm}\left(k_{\rho}\right)=\frac{R_{T E}\left(k_{\rho}\right)}{k_{o z}} \pm \frac{R_{T M}\left(k_{\rho}\right) k_{o z}}{k_{o}^{2}}
$$

where the simple reflection coefficients for a wave going from layer $m$ to layer $n$ are the following

$$
\begin{aligned}
R_{T E}^{m n} & =\frac{k_{m z}-k_{n z}}{k_{m z}+k_{n z}} \\
R_{T M}^{m n} & =\frac{\varepsilon_{r n} k_{m z}-\varepsilon_{r m} k_{n z}}{\varepsilon_{r n} k_{m z}+\varepsilon_{r m} k_{n z}} .
\end{aligned}
$$

The wavenumber is represented by $k_{u}(u=0,1$, or 2$)$ and

$$
k_{u z}=\sqrt{k_{u}^{2}-k_{\rho}^{2}} .
$$

The direct field must be added to the scattered field to obtain the total field. If $z^{\prime}<z$, (44) should be applied.

\section{APPENDIX II}

INTEGRAL SOLUTIONS FOR CASE $2\left(-d<z^{\prime}<0\right.$ AND $\left.z>0\right)$

When the dipole is inside the dielectric layer and the observation point is air, the derivation for the total field is more complicated but is not much different from that of the first case. Simple manipulations of the dyadic Green's function give the transmitted field as

$$
E_{i j}=-\frac{\omega \mu_{o} I_{o} l_{i}}{8 \pi} \int_{0}^{\infty} f_{i j}\left(k_{\rho}\right) e^{i k_{o z} z} d k_{\rho}
$$

where the set of $f_{i j}\left(k_{\rho}\right)$ functions can be shown to be

$$
\begin{aligned}
& f_{z x}\left(k_{\rho}\right)=\frac{-2 i k_{\rho}^{2} k_{o z}}{k_{o}^{2} k_{1 z}}\left[T_{T M}^{\mathrm{up}} e^{i k_{1 z}\left|z^{\prime}\right|}+T_{T M}^{\mathrm{down}} e^{i k_{1 z}\left(2 d+z^{\prime}\right)}\right] \\
& \text { - } J_{1}\left(k_{\rho} \rho\right) \cos \phi \\
& f_{z y}\left(k_{\rho}\right)=\frac{-2 i k_{\rho}^{2} k_{o z}}{k_{o}^{2} k_{1 z}}\left[T_{T M}^{\mathrm{up}} e^{i k_{1 z}\left|z^{\prime}\right|}+T_{T M}^{\mathrm{down}} e^{i k_{1 z}\left(2 d+z^{\prime}\right)}\right] \\
& \text { - } J_{1}\left(k_{\rho} \rho\right) \sin \phi \\
& f_{z z}\left(k_{\rho}\right)=\frac{2 k_{\rho}^{3}}{k_{1 z} k_{o}^{2}}\left[T_{T M}^{\text {up }} e^{i k_{1 z}\left|z^{\prime}\right|}+T_{T M}^{\text {down }} e^{i k_{1 z}\left(2 d+z^{\prime}\right)}\right] \\
& \text { - } J_{0}\left(k_{\rho} \rho\right) \\
& f_{x x, y y}\left(k_{\rho}\right)=k_{\rho}\left\{\left[T_{+}^{\mathrm{up}} e^{i k_{1 z}\left|z^{\prime}\right|}+T_{-}^{\mathrm{down}} e^{i k_{1 z}\left(2 d+z^{\prime}\right)}\right]\right. \\
& \text { - } J_{0}\left(k_{\rho} \rho\right) \\
& \pm\left[T_{-}^{\mathrm{up}} e^{i k_{1 z}\left|z^{\prime}\right|}+T_{+}^{\mathrm{down}} e^{i k_{1 z}\left(2 d+z^{\prime}\right)}\right] \\
& \text { - } \left.J_{2}\left(k_{\rho} \rho\right) \cos (2 \phi)\right\} \\
& f_{x y, y x}\left(k_{\rho}\right)=k_{\rho}\left\{T_{-}^{\mathrm{up}} e^{i k_{1 z}\left|z^{\prime}\right|}+T_{+}^{\text {down }} e^{i k_{1 z}\left(2 d+z^{\prime}\right)}\right\} \\
& \text { - } J_{2}\left(k_{\rho} \rho\right) \sin (2 \phi) \\
& f_{x z}\left(k_{\rho}\right)=\frac{2 i k_{\rho}^{2}}{k_{o}^{2}}\left[-T_{T M}^{\mathrm{up}} e^{i k_{1 z}\left|z^{\prime}\right|}+T_{T M}^{\mathrm{down}} e^{i k_{1 z}\left(2 d+z^{\prime}\right)}\right] \\
& \text { - } J_{1}\left(k_{\rho} \rho\right) \cos \phi \\
& f_{y z}\left(k_{\rho}\right)=\frac{2 i k_{\rho}^{2}}{k_{o}^{2}}\left[-T_{T M}^{\mathrm{up}} e^{i k_{1 z}\left|z^{\prime}\right|}+T_{T M}^{\mathrm{down}} e^{i k_{1 z}\left(2 d+z^{\prime}\right)}\right] \\
& \text { - } J_{1}\left(k_{\rho} \rho\right) \sin \phi \text {. }
\end{aligned}
$$

The various transmission coefficients are defined below

$$
T_{ \pm}^{\mathrm{up}}\left(k_{\rho}\right)=\frac{T_{T E}^{\mathrm{up}}\left(k_{\rho}\right)}{k_{1 z}} \pm \frac{T_{T M}^{\mathrm{up}}\left(k_{\rho}\right) k_{o z}}{k_{o}^{2}}
$$




$$
\begin{aligned}
T_{ \pm}^{\text {down }}\left(k_{\rho}\right) & =\frac{T_{T E}^{\text {down }}\left(k_{\rho}\right)}{k_{1 z}} \pm \frac{T_{T M}^{\text {down }}\left(k_{\rho}\right) k_{o z}}{k_{o}^{2}} \\
T_{P}^{\text {up }} & =T_{P}^{10} \sum_{u=0}^{\infty}\left(R_{P}^{10} R_{P}^{12} e^{i 2 k_{1 z} d}\right)^{u} \\
& =\frac{T_{P}^{10}}{1+R_{P}^{01} R_{P}^{12} e^{i 2 k_{1 z} d}} \\
T_{P}^{\text {down }} & =R_{P}^{12} T_{P}^{10} \sum_{u=0}^{\infty}\left(R_{P}^{10} R_{P}^{12} e^{i 2 k_{1 z} d}\right)^{u} \\
& =\frac{R_{P}^{12} T_{P}^{10}}{1+R_{P}^{01} R_{P}^{12} e^{i 2 k_{1 z} d}} .
\end{aligned}
$$

\section{REFERENCES}

[1] L. M. Brekhovskikh, Waves in Layered Media. New York: Academic, 1960.

[2] L. B. Felsen and N. Marcuvitz, Radiation and Scattering of Waves. Englewood Cliffs, NJ: Prentice-Hall, 1973.

[3] T. Tamir, "Radio waves propagation along mixed paths in forest environments," IEEE Trans. Antennas Propag., vol. AP-25, pp. 471-477, Jul. 1977

[4] L. W. Li, J. H. Koh, T. S. Yeo, M. S. Leong, and P. S. Kooi, "Analysis of radiowave propagation in a four-layered anisotropic forest environment," IEEE Trans. Geosci. Remote Sens., vol. 37, Jul. 1999.

[5] G. P. S. Cavalcante and A. J. Giarola, "Optimization of radio communication in media with three layers," IEEE Trans. Antennas Propag., vol. 31, Jan. 1983.

[6] K. Sarabandi and I. Koh, "Effect of canopy-air interface roughness on HF-VHF wave propagation in forest," IEEE Trans. Antennas Propag., vol. 50, Feb. 2002

[7] W. C. Chew and J. A. Kong, "Asymptotic approximation of waves due to a dipole on a two-layer medium," Radio Sci., vol. 17, May-Jun. 1982.

[8] M. A. Marin and P. H. Pathak, "An asymptotic closed-form representation for the grounded double-layer surface Green's function," IEEE Trans. Antennas Propag., vol. 38, Nov. 1992.

[9] F. T. Ulaby, R. K. Moore, and A. K. Fung, Microwave Remote Sensing. Dedham, MA: Artech, 1986, vol. 3

[10] F. T. Ulaby and M. A. El-Rayes, "Microwave dielectric spectrum of vegetation-Part II: Dual-dispersion model," IEEE Trans. Geosci. Remote Sens., vol. GE-25, Sep. 1987.

[11] M. C. Dobson, F. T. Ulaby, M. T. Hallikaiken, and M. A. El-Rayes, "Microwave dielectric behavior of wet soil-Part II: Dielectric mixing models," IEEE Trans. Geosci. Remote Sens., vol. GE-23, Jan. 1985.

[12] J. E. Hipp, "Soil electromagnetic parameters as functions of frequency, soil density, and soil moisture," Proc. IEEE, vol. 62, Jan. 1974.

[13] M. E. Tiuri, A. H. Sihvola, E. G. Nyfors, and M. T. Hallikaiken, "The complex dielectric constant of snow at microwave frequencies," IEEE J. Ocean. Eng., vol. 9, Dec. 1984.

[14] L. Tsang, J. A. Kong, and R. T. Shin, Theory of Microwave Remote Sensing. New York: Wiley, 1985.

[15] E. C. Jordan and K. G. Balmain, Electromagnetic Waves and Radiating Systems. Englewood Cliffs, NJ: Prentice-Hall, 1968.

[16] J. A. Kong, L. Tsang, and G. Simmons, "Geophysical surface probing with radio-frequency interferometry," IEEE Trans. Antennas Propag., vol. 22, Nov. 1974

[17] W. C. Chew, Waves and Fields in Inhomogeneous Media. New York: Van Nostrand Reinhold, 1990.

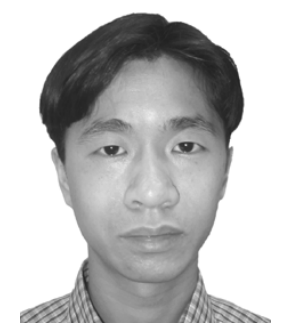

DaHan Liao (S'05) was born in Canton, China. He received the B.S. and M.S. degrees in electrical engineering in 2003 and 2005, respectively, from The University of Michigan, Ann Arbor.

He is currently a Graduate Student Research Assistant with the Radiation Laboratory, The University of Michigan, while also pursuing the Ph.D. degree in applied electromagnetics. His research concentration is on physics-based near-earth wave propagation modeling and simulation.

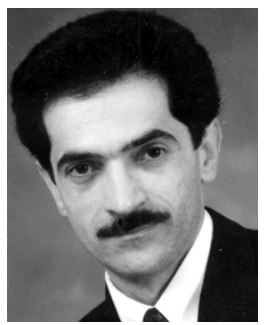

Kamal Sarabandi (S'87-M'90-SM'92-F'00) received the B.S. degree in electrical engineering from Sharif University of Technology, Tehran, Iran, in 1980. He received the M.S. degree, also in electrical engineering, in 1986, the M.S. degree in mathematics, and the Ph.D. degree, again in electrical engineering, from The University of Michigan, Ann Arbor, in 1989

$\mathrm{He}$ is the director of the Radiation Laboratory and a professor with the Department of Electrical Engineering and Computer Science, The University of Michigan. His research areas of interest include microwave and millimeter-wave radar remote sensing, electromagnetic wave propagation, and antenna miniaturization. He has 20 years of experience with wave propagation in random media, communication channel modeling, microwave sensors, and radar systems and is leading a large research group including two research scientists, 10 Ph.D. and 2 M.S. degree students. Over the past 10 years, he has graduated $21 \mathrm{Ph} . \mathrm{D}$. students. He has served as the Principal Investigator on many projects sponsored by NASA, JPL, ARO, ONR, ARL, NSF, DARPA, and numerous industries. He has published many book chapters and more than 120 papers in refereed journals on electromagnetic scattering, random media modeling, wave propagation, antennas, meta-materials, microwave measurement techniques, radar calibration, inverse scattering problems, and microwave sensors. He has also had more than 270 papers and invited presentations in many national and international conferences and symposia on similar subjects.

Dr. Sarabandi is a vice president of the IEEE Geoscience and Remote Sensing Society (GRSS) and a member of the IEEE Technical Activities Board Awards Committee. He is serving as the Associate Editor of the IEEE TRANSACTIONS ON ANTENNAS AND PROPAGATION (AP) and the IEEE Sensors Journal. He is also a member of Commissions F and D of URSI and of The Electromagnetic Academy. He is listed in American Men \& Women of Science Who's Who in America and Who's Who in Science and Engineering. He was the recipient of the Henry Russel Award from the Regent of The University of Michigan (the highest honor the University of Michigan bestows on a faculty member at the assistant or associate level). In 1999 he received a GAAC Distinguished Lecturer Award from the German Federal Ministry for Education, Science, and Technology given to about 10 individuals worldwide in all areas of engineering, science, medicine, and law. He was also a recipient of a 1996 EECS Department Teaching Excellence Award and a 2004 College of Engineering Research Excellence Award. In 2005, he received two prestigious awards, namely, the IEEE Geoscience and Remote Sensing Distinguished Achievement Award and the University of Michigan Faculty Recognition Award. In the past several years, joint papers presented by his students at a number of international symposia (IEEE AP'95,'97,'00,'01,'03, IEEE IGARSS'99,'02, IEEE IMS'01, URSI' 04,'05) have received top student prize paper awards. 